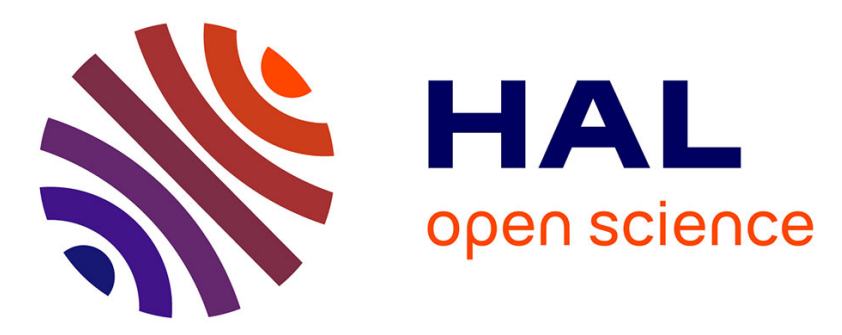

\title{
Espace, temps et fondements : Les mathématiques au défi des autres sciences
}

Giuseppe Longo

\section{To cite this version:}

Giuseppe Longo. Espace, temps et fondements: Les mathématiques au défi des autres sciences. Intellectica - La revue de l'Association pour la Recherche sur les sciences de la Cognition (ARCo), 2003, 1/2 (36-37). hal-03318934

\section{HAL Id: hal-03318934 https://hal-ens.archives-ouvertes.fr/hal-03318934}

Submitted on 11 Aug 2021

HAL is a multi-disciplinary open access archive for the deposit and dissemination of scientific research documents, whether they are published or not. The documents may come from teaching and research institutions in France or abroad, or from public or private research centers.
L'archive ouverte pluridisciplinaire HAL, est destinée au dépôt et à la diffusion de documents scientifiques de niveau recherche, publiés ou non, émanant des établissements d'enseignement et de recherche français ou étrangers, des laboratoires publics ou privés. 
Giuseppe LONGO ${ }^{\square}$

\title{
Espace, temps et fondements : Les mathématiques au défi des autres sciences ${ }^{\curlyvee}$
}

\begin{abstract}
Résumé : L'analyse dominante des fondements des mathématiques ignore dans une large mesure notre relation à l'espace phénoménal. Et, non sans quelque raison. La ruine des présupposés euclidiens, il y a plus d'un siècle, retirant toute certitude à nos "jugements géométriques ", encouragea la recherche des nouvelles " certitudes » arithmétiques, mais contribua à isoler, par là-même, les fondements des mathématiques par rapport aux autres disciplines scientifiques, où la géométrie de l'espace joua un rôle essentiel. Cependant, maintenant que l'approche logique a été couronnée de maints succès, le temps semble venu d'élargir la panoplie d'outils applicables aux recherches fondationnelles en renouant avec les autres sciences. On considérera le rôle de l'espace (et du temps) dans l'organisation du savoir en physique mathématique, en informatique et en biologie. De caractère programmatique, cet essai propose une approche épistémologique du problème des fondements, dont le noyau est une analyse « du processus de connaissance » comme mouvement constituant allant de nos expériences cognitives aux concepts et structures mathématiques.
\end{abstract}

Mots-clés : géométrie, espace et temps, complexité, système cérébral, épistémologie.

Abstract: Space and Time in the Foundations of Mathematics, or some challenges in the interactions with other sciences. Our relation to phenomenal space has been largely disregarded, and with good motivations, in the prevailing foundational analysis of Mathematics. The collapse of Euclidean certitudes, more than a century ago, excluded " geometric judgments" from certainty and contributed, by this, to isolate the foundation of Mathematics from other disciplines, where the relation to space turned out to be crucial. After the success of the logical approach, it is time to broaden our foundational tools and reconstruct, also in that respect, the interactions with other sciences. The way space (and time) organize knowledge is a cross-disciplinary issue that will be briefly examined in Mathematical Physics, Computer Science and Biology. This

$\square$ Laboratoire et Département d'Informatique, CNRS - Ecole Normale Supérieure, 45, Rue d'Ulm, 75005 Paris et CREA, Ecole Polytechnique longo@di.ens.fr ; www.di.ens.fr/users/longo

$\checkmark$ Extrait d'une conférence invitée, First American Math. Soc./SMF meeting, Lyon, July, 2001. Traduit de l'anglais par J.-L. Petit. 
programmatic paper focuses on an epistemological approach to foundations, at the core of which is the analysis of the "knowledge process ", as a constitutive path from cognitive experiences to mathematical concepts and structures.

Keywords: geometry, space and time, complexity, neural system, epistemology.

\section{L'INTELLIGIBILITE GEOMETRIQUE DE L'ESPACE}

«On se gardera de confondre l'évidence primordiale avec l'évidence des axiomes, parce que les axiomes sont eux-mêmes pour la plupart le résultat d'une formation de sens originale et ont toujours déjà cette formation derrière eux $» 1$.

L'homme a, depuis toujours, cherché à organiser l'espace pour lui donner un sens et des structures, par l'action, le geste et le langage. Les mathématiques, plus particulièrement la géométrie, lui ont procuré les plus stables de ses restructurations conceptuelles de l'espace phénoménal. Je vais tenter ici de ressaisir l'unité méthodologique des grandes étapes des mathématiques, qui toutes correspondent à des actes d'unification de l'espace sensible avec l'espace physique ou l'espace mathématique (en ses différentes acceptions). À cette fin, un peu arbitrairement, je soulignerai l'unité du mode de questionnement chez Euclide, Riemann et Alain Connes, en m'attachant moins aux grands noms de ces mathématiciens, ou au détail historique de leurs contributions, qu'aux théories mathématiques qu'ils ont inspirées.

Ce que je soutiens ici, d'une façon fort schématique (parfois presque "apodictique", juste pour proposer un « regard " et une piste de travail), c'est que les diverses façons dont ces trois approches exemplaires rendent l'espace intelligible sont autant de réponses différentes à des questions semblables : quel mode d'accès y avonsnous ? Comment faisons-nous pour le mesurer ? Quels opérateurs pouvons-nous lui appliquer pour agir sur lui ?

\subsection{Euclide}

La géométrie euclidienne organise l'espace à l'aide de figures rigides et des mouvements (rigides) de ces figures. Ces transformations, un groupe, contiennent les homothéties, ce qui fait qu'un théorème, les propriétés d'une figure, demeurent valides pour un élargissement ou une réduction arbitraires des longueurs, surfaces ou volumes de cette figure. D'où une fusion parfaite de l'espace sensible « local », ou « moyen », avec l'espace physique, depuis les très grandes jusqu'aux très petites échelles, propriété qui caractérise la géométrie euclidienne par opposition aux géométries non

\footnotetext{
${ }^{1}$ Husserl, 1933.
} 
euclidiennes. On notera aussi que les postulats d'Euclide sont des constructions: «trace une ligne droite de tel point à tel autre... dessine un cercle de tel centre et tel rayon, etc. " Théorème, en grec, veut dire vue, scène. On montre comment faire des constructions en opérant sur l'espace. (Théorème I, Livre I : «construire un triangle équilatéral sur un segment de droite... chacun sait le faire $»^{2}$ ). En tout cela, on ne se sert que de la règle et du compas, ces " outils» (idéaux) qui rendent l'espace mathématiquement accessible, l'organisent par des figures, que l'on mesure et sur lesquelles on opère. On n'a besoin de rien d'autre.

\section{2 Riemann}

L'objectif principal de Riemann était de rendre compte de la mystérieuse " action à distance » newtonienne. C'est par la " structure de l'espace » qu'il essaie de comprendre la gravitation (mais aussi l'électromagnétisme et la propagation de la chaleur) ${ }^{3}$. Approche révolutionnaire qui est aussi en partie celle de Gauss et Lobatchevski, mais avec Riemann elle est portée à l'unité mathématique la plus haute. Riemann veut comprendre, notamment, les conditions générales de la mesure. Celle-ci requiert la préservation de la rigidité des corps, car c'est en déplaçant un « mètre » rigide qu'on compare des longueurs. L'apport de l'analyse générale des espaces courbes par Riemann a été de montrer que les espaces (ou variétés, multiplicités) à courbure constante garantissent l'invariance de la mesure (la préservation du corps rigide). Les espaces euclidiens ne sont, dès lors, qu'un cas particulier de ces variétés, le cas critique où la courbure est constante et égale à zéro. Mais les autres espaces ont aussi un sens, parce qu'ils peuvent rendre compte de l'action à distance à l'aide de géodésiques. Riemann a l'audace de penser que "les concepts de corps rigide et de rayon lumineux perdent leur validité dans l'infiniment petit »: désormais, les corps n'ont plus besoin d'être rigides, la lumière peut se propager selon des courbes variées... Cela tient au fait - l'un de ses résultats majeurs - que la structure métrique d'une variété (riemanienne), sa mesure par une longueur, peut être mise en corrélation biunivoque avec sa courbure (le tenseur métrique est lié au tenseur de courbure

\footnotetext{
${ }^{2}$ Le point d'intersection des cercles centrés sur les points terminaux ( « les extrémités d'un segment sont des points ») est donné par la continuité dite «parménidienne » des lignes (circulaires), puisque « un point est ce qui est sans parties » et que « deux longueurs sans épaisseur », c.-à-d. deux lignes, engendrent, par leur intersection, " ce qui est sans parties ». De sorte que, chez Euclide, un point est donné comme résultat de l'intersection de deux lignes (comme l'a aussi remarqué Wittgenstein). Seule une réécriture formaliste de la géométrie grecque peut prétendre que ce théorème n'est pas l'objet d'une démonstration rigoureuse de la part d'Euclide (cf. Heath 1908, et un siècle de commentaires).

${ }^{3}$ Riemann, 1854 ; cf. aussi Boi, 1995, Bottazzini et Tazzioli, 1995.
} 
dans les situations qui sont complètement générales). De plus, il peut y avoir un sens à analyser un espace dont la courbure n'est pas constante, dans la mesure où « l'on doit chercher autre part le fondement des relations métriques, dans les forces cohésives agissant sur lui ». De cette idée que les forces entre les corps sont liées à la structure (métrique, locale) de l'espace, H. Weyl dira dans les années vingt, en référence à la théorie de la relativité, qu'elle a été une véritable « divination ». Et que, « ce qui l'autorise, c'est la simplicité de l'explication des phénomènes à laquelle elle conduit ». Avec l'œuvre d'Einstein, nous avons compris l'importance de cette extraordinaire intuition de Riemann.

C'est ainsi que l'organisation géométrique des espaces, à commencer par l'analyse des notions de mesure et de distance, peut faire comprendre les phénomènes physiques, pourvu qu'on généralise la règle et le compas d'Euclide, puisque " dans une variété continue, les relations métriques doivent être introduites sur de nouvelles bases ${ }^{4}$ ». En une authentique "généalogie des concepts mathématiques ", les variétés spatiales explicitent certaines des hypothèses qui fondent l'édifice mathématique sur l'espace phénoménal, dont les plus importantes sont pour Riemann : connectivité, isotropie et continuité $(\mathrm{H}$. Weyl y ajoutera les symétries).

Sans doute, la distinction riemanienne " local - global » (structure métrique et structure topologique, liée celle-ci aux dimensions cartésiennes) nous fait-elle perdre un aspect important de l'approche euclidienne, dans la mesure où l'espace physique (« l'infiniment petit» ou les espaces lointains) peut avoir des propriétés qui échappent à l'expérience sensible. L'expérience « de taille moyenne » n'étant plus transférable à une échelle arbitraire par de simples homothéties, la relation local - global exige une nouvelle mathématique, plus complexe, pour assurer le recollement des cartes locales par des méthodes différentielles. Intuition extrêmement moderne, puisque aussi bien la théorie de la relativité que la physique quantique nous ont appris que l'analyse sensorielle spontanée ne nous donne pas accès au très grand ni au très petit, et qu'on ne saurait donc les mesurer ni opérer sur eux seulement par cette analyse.

Toutefois, les approches de Riemann et d'Euclide n'en conservent pas moins une unité, qui consiste en ce que « faire de la géométrie, c'est étudier les propriétés de nos instruments, c'est à dire du corps rigide ${ }^{5} »$.

\footnotetext{
${ }^{4}$ L'élément linéaire ne se représente plus comme la racine carrée d'une forme différentielle du second ordre (théorème de Pythagore), mais par la formule générale $\mathrm{ds}^{2}=$ $\square g_{\mathrm{ij}} \mathrm{dx}^{\mathrm{i}} \mathrm{dx}$. Cf. citations de Riemann, in Riemann, 1854.

${ }^{5}$ Poincaré, 1913
} 


\subsection{Alain Connes}

Soit un espace $\mathrm{X}$ quelconque, topologique ou métrique, l'ensemble $C(X)$ des fonctions continues de $X$ dans le champ complexe, en tant que structure algébrique (une $\mathrm{C}^{*}$ algèbre commutative), inclut l'espace des mesures sur X. Inversement, d'après un résultat classique de Gelfand, étant donnée $\mathbf{C}$, une $\mathrm{C}^{*}$ algèbre commutative, on peut construire un espace topologique $\mathrm{X}$, tel que $C(X)=\mathbf{C}$. Les points de $X$ seront caractérisés par les idéaux maximaux de $\mathbf{C}$, etc. de manière à reconstruire la structure géométrique de $\mathrm{X}$ sur la base des propriétés de $\mathbf{C}$.

$\mathrm{Si}$ en physique classique, comme en physique relativiste, les mesures sont commutatives, en ce sens que le résultat de plusieurs mesures ne dépend pas de l'ordre dans lequel on les effectue, ce n'est plus le cas en mécanique quantique, où les mesures de la position et du moment (masse $\mathrm{x}$ vitesse) ne sont plus commutatives. Or, ces quantités sont les observables, leur mesure est notre seul accès à « la réalité physique ». Jusque-là, la continuité conceptuelle était complète entre les approches euclidienne et riemanienne. Désormais, " les instruments de mesure » n'ont plus la simplicité relative de la règle et du compas, ni celle de leurs généralisations riemaniennes : " corps rigide », $\left\langle\mathrm{ds}^{2} »\right.$. La mesure requiert les instruments physiques et théoriques complexes de la microphysique, et la mécanique quantique n'a d'autre base de certitude qu'un nombre restreint de phénomènes observables, dont la mesure présente cette non commutativité (qui implique un indéterminisme essentiel). Heisenberg fut le premier à remplacer la mécanique classique par « une mécanique matricielle » dans laquelle les quantités observables ne sont pas nécessairement commutatives. L'algèbre de ses matrices s'obtient à partir d'un groupoïde qui se substitue au groupe (commutatif) classique des mesures. Or, ce dernier groupe est la structure de base d'une reconstruction de l'espace à la Gelfand (d'une algèbre commutative à un espace topologique).

Depuis plusieurs années, l'œuvre du mathématicien Alain Connes est consacrée à reconstruire la géométrie sur la base d'une $C^{*}$ algèbre non commutative en partant d'espaces mesurables pour aller vers des espaces topologiques, métriques et différentiables ${ }^{6}$. Or, l'originalité de ces nouveaux espaces est considérable puisque les notions mêmes de point et de trajectoire ne sont plus les notions classiques ${ }^{7}$. Même si la discussion est encore ouverte, beaucoup de gens pensent que cette approche pourra éclaircir le mystère des phénomènes microphysiques, comme la non localité en vertu de laquelle, en mécanique quantique, une particule n'est pas «localisée

\footnotetext{
${ }^{6}$ Connes, 1994.

${ }^{7}$ Il n'est plus question d'idéaux maximaux, et les trajectoires se rapprochent des « chemins » dérivés de la notion d'intégrale de Feinman.
} 
en un point $»$. En effet, en même temps que la reconstruction de l'espace de la microphysique dépend de l'ordre des mesures, cette dépendance nous donne accès à cet espace. Bref retour à Poincaré (sans prétendre lui attribuer une intuition divinatrice de la possibilité d'une géométrie pour la physique quantique) : "Des êtres qui éprouveraient nos sensations normales dans un ordre anormal, créeraient une géométrie différente de la nôtre ${ }^{8} »$.

\subsection{La géométrie de l'espace physique : remarques épistémologiques}

Partant de ce qui est accessible à la connaissance et la fonde, la géométrie propose une organisation de l'espace physique propre à rendre intelligibles les phénomènes. Or, nous n'avons pas d'autre moyen de constituer une connaissance qu'en commençant par des phénomènes observables et mesurables, cette observabilité dût-elle échapper à notre expérience sensorielle. Comme nous l'ont appris la relativité et la physique quantique, nous pouvons être contraints de renoncer à l'équation " espace sensible = espace physique », qui caractérise les espaces euclidiens avec leur clôture par des homothéties, car nous n'avons pas la règle ni le compas qui nous permettraient d'organiser homothétiquement l'espace des galaxies, ni celui des particules élémentaires. L'intelligibilité à de pareilles échelles ne se fonde plus directement sur nos sens, ni sur l'usage de nos yeux et de nos mains que norment les instruments rigides d'Euclide, mais sur la médiation d'instruments d'observation et de mesure complexes qui dérivent eux-mêmes «d'engagements théoriques » complexes à l'égard de théories ou d'hypothèses fortes, à commencer par la décision même de mesurer « ceci plutôt que cela ».

Notons que toute la différence, ici, est d'ordre cognitif: l'expérience directe des sens ne suffit plus à l'intelligence de l'espace physique ; tandis qu'au plan méthodologique l'unité est sauvegardée. L'engagement ontologique de la géométrie euclidienne en vertu duquel cette géométrie est "l'espace per se » nous a rendus aveugles au fait que l'objectivité mathématique est dans la construction, non dans l'ontologie. Que «l'espace absolu », il n'y a rien de tel. Que ce qu'il y a, c'est la construction objective d'un espace d'action par le sujet connaissant qui emploie les instruments disponibles, et qui atteint l'objectivité dans la mesure où il parvient à relativiser cette construction en se fixant un ou plusieurs systèmes de référence et regards («points de vues »), ainsi que les modalités de communication ou d'interaction entre eux, en spécifiant quels sont ses instruments de mesure. Tandis qu'il n'y a pas une connaissance bien fondée, mais un cadre artificiellement unifié propre à entretenir d'illusoires certitudes, pour un sujet qui croit à des espaces absolus

\footnotetext{
${ }^{8}$ Poincaré, 1902
} 
(Newton), « des lois absolues de la pensée »(Frege), « des vues d'aucun point de vue».

D'Euclide à Riemann et à Connes, en revanche, on aura suivi la bonne méthode, qui consiste à accéder à l'espace, le mesurer et opérer sur lui à l'aide des instruments appropriés qu'on s'est explicitement donnés, en constitutant par le geste organisateur cette construction conceptuelle humaine qu'est la mathématique, en particulier la géométrie. D'un point de vue historique, on rappellera dans le même sens que Poincaré, dans sa critique du logicisme et du formalisme, proposait déjà de compléter la recherche sur les fondements des mathématiques «par une analyse génétique » de leur construction conceptuelle'. Que d'après sa conception de la géométrie comme genèse à partir des mouvements des corps rigides, tant l'approche de Riemann que la référence de Helmholtz «aux faits 》 apparaissent comme des " généalogies de concepts ${ }^{10}$. Enfin, que plutôt qu'empiriste ou rationaliste, Poincaré serait phénoménologue ${ }^{11}$, ou au moins néo-kantien, comme beaucoup de gens l'admettent aujourd'hui ${ }^{12}$. Si les mathématiques se fondent pour lui sur des conventions, celles-ci ne sont pas arbitraires, mais sont plutôt les choix « les plus commodes » pour nous, êtres biologiques, humains, dans ce monde. De sorte que le programme de Poincaré est un essai de fondation des mathématiques sur les régularités du monde tel qu'on le voit. Les structures mathématiques ne sont que des propositions conceptuelles qui visent à rendre le monde intelligible. "Si (la nature) offrait trop de résistance, nous chercherions dans notre arsenal une autre forme qui serait pour elle plus acceptable $e^{13} »$ : cette résistance de la nature s'enracine dans la structure physique et dans notre être biologique, comme dans l'histoire des formations de sens. Pour une version moderne de ce " programme de Poincaré » d'une fondation cognitive des mathématiques, toute la question est de dépasser l'introspection dont dépendaient ces grands mathématiciens (à côté de Poincaré: Riemann, Helmholtz, Enriques, et Weyl) et de mobiliser les ressources des sciences cognitives pour une analyse scientifique de l'action pratique et de la reconstruction conceptuelle du monde ${ }^{14}$.

Le programme fondationnel esquissé ici est-il d'essence épistémologique, dans la mesure où il est une analyse de notre " mode d'accès » à la connaissance et du processus constitutif de celle-ci : en mathématiques, espaces, objets et structures sont donc construits sur la base de l'admission expresse de leur fondement

\footnotetext{
${ }^{9}$ Heinzmann, 1998

${ }^{10}$ Nabonnand, 2001

${ }^{11}$ Husserl, 1933.

${ }^{12} \mathrm{Cf}$. pour des références bibliographiques : Nabonnand, 2000.

${ }^{13}$ Poincaré, 1908.

${ }^{14}$ Cf. notre manifeste sur le réseau, Longo et al., 1999 et Longo, 2001.
} 
cognitif, et cela est objectif. Sans doute, ce type d'analyse a-t-il été écarté au $\mathrm{XX}^{\text {ème }} \mathrm{s}$. par le programme formaliste et logiciste de fondement des mathématiques, qui ne s'intéressait qu'aux démonstrations (formelles). Mais, si nécessaire qu'ait été cette investigation, elle a cru pouvoir faire l'économie d'une analyse de la constitution des concepts et des structures en encodant le monde dans une suite de signes sans signification. Et, si abstraites et symboliques que soient les mathématiques, leur identification séculaire avec le formel a privé l'analyse fondationnelle de ces dimensions de signification et d'épistémologie qu'il importe de lui restituer en étendant le projet fondationnel au "cheminement constituant » des structures mathématiques abstraites à partir de leurs origines signifiantes dans l'espace et le temps phénoménaux (et leur mode d'organisation).

\section{CODAGES}

Il n'est pas facile de se faire une idée de la gravité de la crise des certitudes euclidiennes vieilles de 2.300 ans que l'avènement des approches non euclidiennes a provoquée au XIX ${ }^{\text {eme }} \mathrm{s}$. Les investigations de Frege ont inauguré la moderne « issue royale » à ce nouveau problème de l'espace, qui fait de la logique (mathématique) la solution de rechange à une analyse fondationnelle sur la base de l'espace phénoménal. «Aux axiomes de la géométrie, se conforment les visions les plus sauvages du délire, dans la mesure où elles restent des intuitions " ; de sorte que si l'on veut une certitude absolue, on ne pourra l'avoir qu'en référence au concept de nombre et aux lois logiques qui le régissent : « les vérités arithmétiques règnent sur le domaine du dénombrable. C'est le plus étendu, car en fait partie non seulement l'actuel, non seulement l'intuitionnable, mais tout le pensable $^{15}$ ». La géométrie elle-même (mais Frege, par prudence, ne considère que la géométrie euclidienne) peut être fondée analytiquement sur la notion de nombre, en tant que relation entre longueurs ${ }^{16}$

Ce programme fut réalisé ultérieurement (quoique différemment) par Hilbert, qui dès son premier ouvrage sur les fondements a réduit le problème à une analyse de la consistance formelle, c'est-à-dire à la non contradiction des axiomes des mathématiques, et en particulier de la géométrie, sans référence à la signification, et plus spécialement à l'espace ${ }^{17}$. Sa prouesse technique a été de donner toutes les preuves de « consistance relative » en géométrie : poser un axiome, en supprimer un (euclidien, non euclidien, désarguien, non désarguien, archimédien, non archimédien...)... inclure un système formel dans un autre. Au-delà de l'œuvre de Beltrami et Klein, les

\footnotetext{
${ }^{15}$ Frege, 1884, § 14, p. 20.

${ }^{16}$ Frege, 1873, p. 9-10. Cf. discussion in Tappenden, 1995.

${ }^{17}$ Hilbert, 1899.
} 
interprétations relatives des espaces de Lobatchevski et de Riemann en termes d'espaces euclidiens sont élevées par là au plus haut niveau de rigueur et de généralité. Enfin, le chef-d'œuvre : avoir formellement encodé, par des outils analytiques, la géométrie euclidienne dans l'arithmétique. L'année suivante, avec la formulation du problème de la consistance de l'arithmétique à la conférence de Paris, était mis en place le programme scientifique du fondement formel : sans aucune référence à la signification ni à l'espace, ni à notre mode d'accès à celui-ci, se contenter de démontrer formellement que les axiomes de l'arithmétique n'implique pas que " $0=1$ ». Problème fondationnel des mathématiques qui inclut celui de la géométrie, puisque celle-ci, par encodage (immersion conservative), n'est qu'un sous-système de l'arithmétique.

Nombreux sont ceux qui font grand cas de cet extraordinaire tour de force of Hilbert. Poincaré, lui-même, qui dans son compte rendu de l'ouvrage de Hilbert de 1899 reconnaît la performance technique, mais n'en souligne pas moins la déperdition de signification, la trivialisation de notre conception de l'espace, l'absurdité d'une référence aux mathématiques comme codage d'axiomes par «le piano raisonneur de Stanley Jevons » d'où « on verrait sortir toute la Géométrie ». Ailleurs, Poincaré se réfère à la conception des mathématiques qui sous-tend les programmes fondationnels de Peano, Padoa, et Hilbert, comme à "la machine à saucisses de Chicago " : avec des cochons et des axiomes, produire des saucisses et des théorèmes ${ }^{18}$.

Centré sur l'arithmétique, lieu théorique des codages, le programme fondationnel de Hilbert a inauguré une bonne moitié du $\mathrm{XX}^{\mathrm{eme}}$ s., qu'on a fort justement appelé « le temps des codages ${ }^{19}$ ". En est issu le codage de la connaissance comme déduction formelle, encodé à son tour comme programme et exécuté par machine numérique (machine de Turing). Distinguant entre matériel et logiciel (programme) Turing mettait en route une " théorie des programmes ». La machine de Turing universelle qui encode n'importe quelle autre machine de Turing et en simule les opérations engendrait le système d'exploitation et le compilateur: Poincaré n'imaginait pas que « la machine à saucisses » aille aussi loin. Mais, ainsi va l'histoire : de mauvais programmes fondationnels qui reposent sur de conjectures fausses (décidabilité formelle, complétude et consistance finiment démontrable de l'arithmétique), pourvu qu'elles soient précises et robustes, résultent les plus grands profits. De même, "l'analyse des perturbations planétaires 》 de Laplace, censée rendre compte complètement du futur (et du passé) de systèmes déterministes régis par les lois de Newton, a-t-elle été,

\footnotetext{
${ }^{18}$ Bottazzini, 1999.

${ }^{19}$ Girard, 2001.
} 
en dépit de son caractère irréalisable démontré par Poincaré $^{20}$, à l'origine des travaux considérables en analyse réalisés au XIX ${ }^{\text {èmé }} \mathrm{s}$. (analyse des perturbations planétaires).

Vient le temps, néanmoins, de la critique des mauvaises idées. Par exemple, la clé du programme fondationnel de la logique mathématique est la croyance en "la transparence" des codages, le contenu d'information devant, suppose-t-on, être préservé par tout codage "raisonnable ». Prendre n'importe quel fragment des mathématiques, l'encoder dans les axiomes de la théorie des ensembles (ou, mieux, dans l'arithmétique), démontrer la consistance relative du système en question, et le tour est joué : grâce à un codage $0-1$, on peut l'entrer dans une machine de Turing ou un ordinateur. Que cette réduction soit rarement conduite jusqu'au bout ne l'empêche pas d'avoir un énorme impact en théorie des ensembles et théorie de la démonstration ${ }^{21}$.

Sans disputer à l'analyse logique formelle son rôle de composante nécessaire de la recherche fondationnelle en mathématique et informatique, il nous incombe à présent d'enrichir le programme de cette recherche en lui procurant ce qui lui manque : sens et signification, référence à l'espace, comme question de cognition et comme lieu des phénomènes physiques. Or, il se trouve que la même théorie de la démonstration qui a donné naissance au paradigme dominant (l'information et la déduction indépendante de la signification et du codage) est en train de s'ouvrir à de nouvelles suggestions. Le logicien Jean-Yves Girard ${ }^{22}$ a proposé une méthode d'analyse des démonstrations « sensible au codage », où la distinction syntaxe - sémantique est privée de sens ${ }^{23}$. Dans ce cadre nouveau, la structure géométrique des démonstrations est prise en compte dans la conduite de celles-ci. Leurs propriétés de connectivité (au sens de Riemann) et de symétrie (au sens de Weyl) leur tiennent lieu de " codage géométrique » nonobstant le fait que Riemann et Weyl pensaient plutôt à des espaces physiques, parce que

\footnotetext{
${ }^{20}$ Poincaré, 1908.

${ }^{21}$ Asperti et Longo, 1991, Longo, 2002. Les historiens nous disent que Hilbert n'était pas formaliste, et il est vrai qu'il n'était pas indifférent aux structures, ni à la signification physique, en mathématiques. Toutefois, la perspective technique de son ouvrage de 1899 , et de là, le programme fondationnel défini entre 1900 et les années 20, est devenue le paradigme du formalisme qui a grevé le siècle dernier de l'hypothèque d'une analyse incomplète des fondements. On a tenté de réactiver ce programme de Hilbert, mais c'est plutôt celui de Poincaré qu'il faudrait réactiver pour comprendre la pertinence des mathématiques pour la connaissance de l'espace et la cognition.

${ }^{22}$ Girard, 2001

${ }^{23}$ La définition très générale de " géométrique » comme "sensible aux codages » est le résultat d'une longue et passionnante discussion avec J.Y. Girard à la terrasse ensoleillée d'un café à Marseille, en avril 2001, sur ce que " géométrique » voulait dire et qui était commun, dans nos différents projets scientifiques.
} 
dans les systèmes de Girard tout se passe comme si ces propriétés spatiales, qu'on avait chassées par la porte, réintégraient la construction des démonstrations en passant par la fenêtre. Pour Poincaré, déjà, les prémisses se rattachaient à la conclusion au moyen d'une «architecture mathématique » et que dans son combat contre le formalisme le même Poincaré insistait sur la non invariance du raisonnement mathématique par rapport à la signification ${ }^{24}$, montrant une remarquable intuition de l'incomplétude des formalismes ${ }^{25}$.

\section{LA GEOMETRIE DANS LE CALCUL}

Filles directes du tournant logico-linguistique, les machines de Turing ne sont pas dans l'espace et génèrent seulement un temps absolu (newtonien). Que leur architecture (tête de lecture et bande) soit à une, deux ou $\mathrm{n}$ dimensions ne change rien à leur capacité d'expression, parce que la dimension cartésienne de leur processus physique n'affecte pas leurs calculs. Ce qui tient évidemment au fait que ces "machines " sont des constructions purement logiques issues d'une réduction de la notion de déduction séquentielle à des pas élémentaires (déplacer la tête à droite ou à gauche, écrire 0 ou 1 sur la bande). Ici, plus de physique, l'espace ayant depuis longtemps été exclu de la discussion sur les fondements. A-t-on jamais vu un processus physique indépendant des dimensions ? En certains cas, depuis la relativité, et plus encore pour la physique quantique en théorie des cordes, il semble, au contraire, qu'il n'y ait que les dimensions qui comptent.

Pour ce qui est du temps dans les machines de Turing, il n'est pas seulement absolu et linéaire, mais généré par l'horloge, à l'opposé de ce qui se passe en physique, où la conception relationnelle du temps s'est imposée avec l'abandon des absolus newtoniens. Mesurer le temps avec l'horloge solitaire d'une machine de Turing, c'est avoir un mètre dans un univers vide : dans cet univers où il n'y a aucune distance, mais seulement ce mètre.

En un mot, les machines de Turing sont des machines logiques (Logical Computing Machine, dans la définition de Turing) non des machines physiques ${ }^{26}$. La théorie de la calculabilité des années 30 est à l'origine de l'informatique avec ses styles de programmation (logique, impérative, fonctionnelle), art de faire fonctionner des machines en un sens purement logique. Le logiciel, dont la

\footnotetext{
${ }^{24}$ Poincaré, 1905, 1908. Cf. Heinzmann, 1998.

${ }^{25}$ Cf. également Weyl, 1918, pour sa conjecture au sujet de l'incomplétude de l'arithmétique. Cf. Longo, 1999, sur le fait que la démonstration des jugements formellement indémontrables de l'arithmétique réintroduit la signification et, Longo (2002), des « jugements géométriques ».

${ }^{26}$ Longo, 2002a.
} 
distinction d'avec le matériel fonde la science de la programmation, aura été conçu (jusqu'au défi de la programmation concurrente) d'après le paradigme de Turing et ses contemporains ${ }^{27}$. La physique qui prend sa source dans les problèmes de l'espace et du temps, échappe à ces styles de programmation, ce qui les rend inadéquats (sauf extension significative) pour les systèmes distribués, asynchrones et concurrents.

Outre l'intelligibilité géométrique de l'espace, le $X^{\text {ème }}$ s. a procédé à une analyse approfondie du temps physique. Le temps relativisé, mais réversible, de la relativité, le temps irréversible avec bifurcations des systèmes dynamiques (en physique des états thermodynamiques ou critiques), le temps plus complexe encore de la physique quantique, aucune de ces formes de temps ne renvoie à une horloge unique et absolue ; au contraire, le temps est plutôt pour elles un problème de synchronisation entre systèmes éventuellement asynchrones. Or, ces dernières décennies, les machines, ces mêmes calculatrices numériques sorties de la tête de Turing (et Peano, et Hilbert), ont été distribuées dans l'espace par les ingénieurs, qui ont eu l'audace d'en mettre plusieurs en concurrence dans un même calcul, de sorte que des processus qui peuvent être très distants ne sont plus isolés dans le vide, mais se déroulent parallèlement, entrent en communication entre eux et ont accès à la même base de données. Cette concurrence résume la forme nouvelle de notre problème, qui tient moins au parallélisme des calculs qu'à la communication et au partage entre eux des données et programmes, chaque processus pouvant éventuellement avoir sa propre horloge.

Première différence, les calculs cessent d'être compositionnels, tandis que toute la théorie de la calculabilité, qui date des années 30, repose sur la compositionnalité, et que la théorie des fonctions récursives de Herbrand, Gödel et Kleene, le lambda calcul Church ${ }^{28}$, et bien sûr les machines de Turing (théorie computationnellement équivalentes) s'obtiennent par «composition » d'un petit nombre de fonctions (respectivement, termes, et pas) de base. Leur " sémantique mathématique » étant compositionnelle, l'analyse de la fonction à calculer peut se faire morceau par morceau dans la perspective d'une composition ultérieure. En revanche, un calcul effectué par des processus concurrents ne se laisse pas analyser (on n'en connaît pas la sémantique, on ne peut dire quelle fonction est calculée) en procédant d'abord à l'analyse de chaque processus individuel pour passer ensuite à la composition des résultats obtenus, parce que ces processus interagissent les uns avec les autres au cours

\footnotetext{
27 «La programmation impérative » a son origine dans le travail de Turing, « la programmation fonctionnelle », dans le 0 Calcul de Church, « la programmation logique », dans le théorème d'Herbrand.

${ }^{28}$ Longtemps centre d'intérêt de l'auteur de cet article, avec sa très riche "sémantique catégorique » (voir Asperti et Longo, 1991).
} 
du calcul, et ce d'autant plus qu'ils entrent en compétition pour avoir accès à un même base de données, et que l'usage de celle-ci par l'un des processus peut la changer.

Imaginez un système de réservation de compagnie d'aviation: dans un tel système distribué l'important est l'ordre de priorité et la synchronisation de l'accès à une base de données en changement constant (au moment où un agent modifie cette base de données les autres ne doivent pas y avoir accès). Imaginez, maintenant, deux processus, $x$ et $y$. Dans un système séquentiel vous pouvez avoir « $x$ puis $y$ » ou "y puis $x$ », qui s'excluent mutuellement et excluent toute autre possibilité. Soit le rectangle dont les côtés sont nommés $x$ et $y$; les deux chemins séquentiels ci-dessus sont la composition des côtés le long des bords, ils vont successivement du coin d'en bas à gauche $(0,0)$, au coin d'en haut à droite $(1,1), p$. ex. Mais, si les deux processus interagissent pendant le calcul, et/ou ont accès à la même base de données, on n'aura une bonne représentation des calculs possibles qu'en prenant tous les chemins (de dérivée positive) dans le rectangle qui va de $(0,0)$ à $(1,1)$. Les situations d'inaccessibilité peuvent être représentées par des « trous » à l'intérieur du rectangle : quand un processus passe par un certain statut ou région, un autre ne peut pas agir. L'existence d'un ou plusieurs trous permet de classer les chemins par " classes d'homotopie », une même classe contenant les chemins tels qu'on passe de l'un à l'autre par une déformation continue, ou qui se transforment les uns dans les autres sans avoir à franchir un trou. Mathématique non triviale, la théorie de cette homotopie traite de la connectivité spatio-temporelle, ou équivalence sous une déformation dans des variétés à $n$ dimensions (autant que de processus $)^{29}$. La différence avec la géométrie standard, c'est l'omniprésence de l'irréversibilité du temps, un temps qui ne peut plus être linéaire, mais qui se ramifie à la façon des bifurcations des systèmes dynamiques. Sa représentation requiert qu'on paramétrise les chemins en fonction du temps, et qu'on admette que ces chemins sont des fonctions croissantes de $x$ et de $y$, des " chemins dirigés ", nouvelle problématique non triviale de l'analyse homotopique. Enfin, " effectuable » prend un autre sens qu'en calculabilité classique, où l'on peut (p. ex.) démontrer que le problème de l'arrêt est indécidable et qu'il existe des calculs partiels qu'on ne peut pas étendre à un calcul total. Désormais, il s'agit plutôt de savoir si tel calcul est (un chemin) accessible comme élément d'une classe d'homotopie donnée dans une certaine variété à $n$ dimensions. Des méthodes purement topologiques donnent des résultats d'impossibilité avec notamment des bornes inférieures de temps ${ }^{30}$.

\footnotetext{
${ }^{29}$ À ces nouveaux secteurs de l'informatique, on est surpris de voir s'appliquer des travaux de Serre en géométrie pure et géométrie algébrique, cf. Goubault, 2000.

${ }^{30}$ Goubault, 2000.
} 
Il n'y a, bien sûr, aucun sens à vouloir coder tout cela dans des machines de Turing, parce que des systèmes concurrents ne traitent pas uniquement des suites de 0 et de 1 , mais qu'ils ont un flux d'entrée et de sortie, qu'ils sont ouverts aux interactions avec l'environnement, sans parler du problème complexe du temps, relationnel et arborescent. Leur simulation par machines séquentielles requiert la construction de quotients sur les chemins de calcul, une " simulation modulo » qui est loin d'être standardisée ou unique, mais qui est souvent ad hoc et passe à côté du défi que constitue le calcul concurrent ${ }^{31}$. Par exemple, dans ces systèmes, plus que la relation d'entrée - sortie, c'est le mode d'évolution d'un calcul dans l'espace et le temps qui compte. En guise « d'observable » on a ici des structures spatio-temporelles; ils posent de nouvelles questions et procurent de nouvelles intuitions. De nombreux problèmes restent ouverts, dont celui des systèmes (distribués, non séquentiels) admettant des défauts tolérables, à savoir de (petites) déformations continues à l'intérieur d'une classe d'homotopie ; celui de la synchronisation : un système est «distribué » quand le temps requis pour faire communiquer les processus est du même ordre de grandeur que le pas élémentaire du calcul dans un processus. Ce dernier étant aujourd'hui d'une nanoseconde, laps de temps pendant lequel la lumière ne fait que $30 \mathrm{~cm}$, la synchronisation d'un système concurrent qui serait distribué à la surface de la Terre (donc dans des systèmes d'accélérations différents) pourra se heurter à des problèmes relativistes, avec toutes les difficultés de calcul des délais relativistes ${ }^{32}$

Concluons que même les calculateurs numériques, une fois qu'on les a réintégrés à l'espace physique, sont loin de réaliser le projet fonctionnaliste, d'après lequel une machine de Turing, pourvu qu'on y encode le monde, représentera n'importe quel système physique, y compris les systèmes biologiques, bien entendu. Il suffit de prendre en compte la distribution dans l'espace de ces mêmes machines pour que changent, avant même les réponses, les questions à poser au système physique qu'on veut comprendre.

\section{VIVRE DANS L'ESPACE ET LE TEMPS}

Pour nous, les phénomènes cognitifs appartiennent au vivant, domaine qui inclut de la cellule à l'homme. D'autres peuvent s'intéresser à la cognition pour des entités immatérielles sur Sirius, ou encore à des ordinateurs de divers types : on change de sujet. Le cerveau est, nous l'admettons, une machine matérielle, mais vivante,

\footnotetext{
${ }^{31}$ Aceto et al., 2003.

${ }^{32}$ Présentant un point de vue fondé sur des résultats spécifiques, cette section (et cet article) ne mentionne pas les nombreuses autres approches du calcul concurrent qui font intervenir l'espace d'une manière différente et qui restent des variantes de l'analyse du calcul classique. Cf. Bahsoun et al., 1999, Aceto et al., 2002.
} 
qui chez les humains ne vit que dans son écosystème privilégié, qui est le crâne d'un homme vivant dans l'histoire (la mémoire commune d'une communauté communicante). Si l'on peut, sporadiquement, isoler certaines performances cognitives et les transférer à des machines, cela ne change rien au fait que la cognition humaine dépend de la vie, ce qui ne veut pas dire qu'elle se réduise à la biologie comme science, parce qu'elle dépend aussi du langage et de l'histoire. Notre connaissance du vivant ne saurait, avec son caractère hautement évolutif, nous procurer une explication complète de phénomènes qui ont requis jusqu'à maintenant des méthodes aussi différentes que celles des sciences de la communication et des sciences historiques. S'élever à une synthèse nouvelle est le défi que les sciences cognitives ont à relever aujourd'hui.

Les phénomènes du vivant mettent également la mathématique au défi par leur caractère spatio-temporel, puisque depuis la structure tridimensionnelle de l'ADN et les protéines (briques élémentaires du vivant, sinon vivantes elles-mêmes) qui se plient et se déplient, tout processus vital est une dynamique de formes. De cette organisation spatiale, il faut tenir compte pour comprendre la complexité des structures du vivant. Leur fonctionnalité est manquée par un encodage linéaire comme celui d'une description sur la bande d'une machine de Turing ${ }^{33}$. De même, nous tenons que toutes les fonctions cognitives sont des épiphénomènes irréductibles de la vie.

Revenons à la théorie classique de la calculabilité pour avoir un terme de comparaison avec la complexité des phénomènes de la vie et de la cognition. Quelle que soit la capacité expressive de cette théorie son caractère compositionnel lui ferme les systèmes non compositionnels, dont les plus intéressants pour notre propos sont les systèmes dynamiques. Exemple. Le mouvement de corps planétaires obéit aux lois de la gravitation de Newton. Considérons indépendamment deux systèmes de deux corps : ces deux systèmes indépendants se stabilisent en orbites périodiques, comme l'a prédit Képler. Mais, si vous mettez ensemble les quatre corps, d'après la célèbre analyse de Poincaré, il s'ensuit une organisation spatiale inédite, un comportement chaotique, tel que le système formé par ces quatre corps ne peut pas être considéré comme la composition des deux systèmes de deux corps. En conclusion, il suffit de passer de la machine de Turing à bande unidimensionnelle, ou tout système de règles formelles équivalent, à des systèmes distribués concurrents de calcul ou à des systèmes gravitationnels un peu riches, pour avoir besoin d'une mathématique entièrement nouvelle.

Cette confrontation avec quelques ordinateurs ou corps gravitationnels suffit pour faire justice du mythe fonctionnaliste de l'indépendance des codages et des structures prêtées aux

${ }^{33}$ Cf. supra $\S .2 .1$. 
phénomènes cognitifs, expression la plus complexe de la vie. Si un phénomène artificiel ou naturel nécessite pour sa représentation l'une des approches non classiques ci-dessus, c'est qu'il est irréductible à un système linéaire, compositionnel et non spatial comme la machine de Turing. Toutefois, même s'il y a sûrement du calcul concurrentiel et de la dynamique physique dans le vivant, il faut faire encore un pas de plus dans le sens de la complexité conceptuelle pour accéder aux mathématiques requises pour sa représentation, à supposer qu'elles soient possibles. Un petit nombre de lois sur un seul niveau conceptuel suffit pour décrire tous ces «systèmes uni-échelle », processus qui n'interagissent que par des signaux numériques, des forces gravitationnelles, ou d'autres « structures de réseau » d'un type conceptuellement similaire. En ce qui les concerne, les mathématiques sont tout à fait efficaces (bien qu'on manque encore d'une bonne théorie du calcul concurrent ou de la dynamique des fortes turbulences : les équations de Navier-Stokes ne décrivent convenablement les flux que loin des bords, où les turbulences sont maximales ${ }^{34}$.

Or, les phénomènes biologiques sont, quant à eux, multi-échelles. Ils le sont essentiellement, et pas seulement en apparence comme la mathématique des fractales, où partant d'un niveau de grandeur donné on peut progresser vers des phénomènes de plus en plus fins en passant à d'autres échelles, mais en appliquant toujours la même loi unique, indéfiniment itérée. Sans doute, quelques entités vivantes ou composantes se développent-elles de la sorte : les fractales donnent des systèmes vasculaire et respiratoire des descriptions fort convaincantes en terme d'une loi mathématique de maximation des surfaces d'échange et des volumes d'irrigation ${ }^{35}$. Mais, dans ces situations particulières, la vie ne se caractérise que par un facteur de croissance qui ressortit de la même analyse purement physique que la cire dans une ruche. Le vivant est, à notre sens, une hiérarchie où alternent deux niveaux d'organisation, au moins : celui des individus biologiques autonomes (cellules) et celui des groupes organisés d'individus (organes), lesquels, à leur tour, s'intègrent par leur fonction biologique à un niveau d'unité supérieure (et donnent une nouvelle unité vivante). $\mathrm{Si}$, en termes physico-mathématiques, on retrouve dans les organes des géométries fractales, les interactions entre unités biologiques seraient plutôt associées à des systèmes dynamiques $^{36}$. Notons qu'on trouve également des organes à l'intérieur des cellules : la membrane externe et le cytosquelette, mais aussi des espèces de membranes ou rails interne, les microtubules, qui jouent un rôle clé dans le métabolisme et la reproduction de la cellule. Cet aperçu n'est que le début d'une conception de la complexité appliquant des outils mathématiques

\footnotetext{
${ }^{34}$ Farge et al., 1996.

${ }^{35}$ Brown, 1999 ; Nonnenmacher, 1994 ; Bailly et al., 1991.

${ }^{36}$ Bailly et al., 1993, Bailly et Longo, 2003.
} 
usuels déjà divisés en différents cadres théoriques en fonction de l'échelle considérée, outils qui prétendent chacun à une certaine complétude descriptive, mais seulement à son propre niveau d'investigation. De plus, ces deux niveaux (schématiques) interagissent verticalement, et donnent lieu à un nouveau système multi-échelle, tel que tout changement d'échelle change la mathématique employée à sa description.

\section{PHENOMENES MULTI-ECHELles ET COMPLEXITE DU SYSTEME NERVEUX}

Quand on considère le cerveau et ses fonctions, notre objet de connaissance le plus complexe, la situation devient encore plus riche. Les neurones communiquent entre eux : à un premier niveau, ils échangent des neurotransmetteurs de composition chimique variée. Leur fonctionnalité dépend aussi de la forme des récepteurs postsynaptiques, protéines complexes dont la forme externe et les canaux internes déterminent le transfert d'ions dans les neurones récepteurs. Ensuite de quoi se produit une cascade d'événements biochimiques où les protéines jouent un rôle complexe de transmission et de facilitation ou inhibition de l'activation subséquente des récepteurs. Notons que, dans ces protéines, éléments de base de la vie, la fonction est dans la forme, car ces grosses molécules interagissent dans les échanges métaboliques et les échanges d'informations en fonction de leur repliement tridimensionnel.

Passant à une échelle supérieure, celle des neurones comme touts, on a des entités à six dimensions : trois dimensions d'espace, plus trois autres pour la forme de leur profil de réponse. Traiter ces neurones comme simples « éléments à seuil » est une approximation grossière, car si ces seuils sont effectivement importants pour la communication neuronale, c'est à la façon de l'onde porteuse des télécommunications, et cela ne doit pas nous faire oublier que la structure géométrique fine et la modulation du profil d'activation d'un neurone contribuent aussi à l'élaboration de cette information.

Exemple: les neurones du cortex visuel V1. Leur profil de réponse présente la forme très particulière d'une gaussienne asymétrique étendue sur un rectangle (ou plutôt une dérivée de gaussienne, puisqu'on observe une sorte de " pente de Mach » qui accentue les contrastes). Ce rectangle indique la direction du bord détecté dans le champ récepteur. V1 donne ainsi l'orientation locale du bord (virtuel) d'un objet du champ visuel, comme par une " dérivée » le long d'une courbe. Après quoi V1 "intègre », ou " recolle " toutes ces cartes locales unidimensionnelles grâce à la connectivité complexe des neurones iso-orientés ${ }^{37}$. Dans ces deux activités, le rôle du profil d'activité semble déterminant.

${ }^{37}$ Petitot, 2000 ; Gilbert, 1992. 
À une échelle encore supérieure, on a les réseaux neuronaux, qu'on étudie à l'aide de la géométrie des systèmes dynamiques ${ }^{38}$, de là : les assemblées de réseaux, assemblées d'assemblées, etc., qui font l'objet d'analyses de la complexité et de la synchronisation de leur fonctionnalité39. Bien qu'on prétende que c'est à ce dernier niveau qu'apparaissent les fonctions cognitives conscientes, toutes ces structures de cet « emboîtement » concourent à l'élaboration de l'information, qui, en grande partie, est affaire de géométrie. Depuis le repliement spatial des récepteurs post-synaptiques et des protéines dans les cascades biochimiques subséquentes à l'intérieur des neurones, jusqu'à la forme du profil de réponse de ceux-ci, et à la synchronisation des réseaux, puis des assemblées, tous ces niveaux contribuent et interagissent, à la fois horizontalement et verticalement : un état psychologique peut affecter la fonctionnalité de certains neurotransmetteurs au niveau inférieur, et vice versa (les psycho-médecines agissent au niveau des neurotransmetteurs et des neurorécepteurs).

Nous avons là un défi majeur pour les mathématiques, qui ont à inventer les outils adéquats à ces systèmes multi-échelles inhérents à la vie. Toutes les tentatives d'isolement d'un niveau particulier (la fonction logique pure, l'analyse fine des formes dynamiques de connectivité, etc.), si utiles qu'elles soient, demeurent essentiellement incomplètes comme approches mathématiques des fonctions cérébrales et cognitives.

\section{FORMES GEOMETRIQUES ET SIGNIFICATION}

Pour la vie, formes et changements de formes sont signifiants, les formes et leur interaction dans l'espace contribuent à la signification. Une cellule se donne à elle-même une forme ou se meut dans l'espace pour préserver ou améliorer son métabolisme ou pour se reproduire. Pour elle, un signal entrant ou un choc physique sont signifiants. La signification du signal tient à la façon dont il affecte ses déformation ou mouvement orientés vers un but, son action. Et les neurones, en tant que cellules, ont une forme à six dimensions, compte tenu de leur profil de réponse électrostatique. De sorte qu'un signal, y compris électrique, est signifiant pour un neurone en fonction du mode de participation de ce signal à son métabolisme par la déformation qu'il induit. De là, la signification affecte les réseaux de neurones, assemblées de réseaux, etc., selon leurs formes spatiotemporelles, et leurs variations. De constitution non compositionnelle, cette signification - de même que la signification contextuelle d'une expression linguistique, $\mathrm{si}$ riche d'intersubjectivité et d'histoire - ne saurait se laisser réduire à l'activité géométrique d'un réseau neuronal. Pour des entités

\footnotetext{
${ }^{38}$ Hertz et al., 1991.

${ }^{39}$ Edelman, 2000 ; Varela, 1999.
} 
vivantes complexes, la signification est dans la relation entre une activité neuronale (comme forme évolutive d'un réseau) et son contexte de vie. Impossible de reconstruire cette signification par la seule lecture d'une activation ou d'une déformation sans considérer en même temps l'écosystème et, pour les humains, leur expérience intersubjective et historique de cet écosystème. La signification est une question de relation et d'interaction entre des termes qui incluent des entités vivantes et leurs formes. Toutes ces formes vivantes et leurs variations sont porteuses de signification, une signification que co-constituent en leur interaction ces mêmes formes évolutives et leur contexte. C'est là que la signification prend sa source. Ultérieurement les diverses significations s'organisent à différentes échelles, jusqu'à atteindre la richesse de notre communauté humaine communicante.

Le défi, pour les sciences cognitives, est qu'il leur faut circuler d'une échelle à l'autre sans prétendre réduire les unes aux autres, mais en comparant et en faisant interagir les différentes méthodes ajustées aux différentes phénoménalités et différents niveaux, depuis la cellule jusqu'à l'histoire. La tâche est de réaliser une synthèse nouvelle, ce qu'on n'a jamais pu faire par le passé avec un simple transfert de techniques. Sans doute, la séparation artificielle entre les formalismes déductifs et la sémantique nous a-t-elle apporté les ordinateurs et leurs langages de programmation, mais dans ce cas le problème se pose de comment des chaînes de nombres binaires peuvent être porteuses de signification. Les chaînes de 0 et de 1 des langages formels doivent être décodées et interprétées (compilées) : problème de programmation et de sa théorie. En revanche, les êtres vivants, quand ils élaborent ou transmettent des significations, des harmonies, des émotions, etc. induisent des déformations dans leurs systèmes neuronaux qui véhiculent ces contenus dans leur structuration géométrique et ses variations. De sorte que le défi de la cognition, si on associe la signification et l'information aux déformations du vivant, à leurs géométries évolutives, est de comprendre la co-constitution non compositionnelle du sens, des entités vivantes élémentaires aux êtres historiques, comme une interaction enchevêtrée de phénomènes. Le caractère multi-échelle de ce processus est un défi majeur pour les mathématiques.

\section{CONCLUSION EPISTEMOLOGICO-MATHEMATIQUE}

Dans l'esprit de cet article, j'indiquerai maintenant certaines directions possibles d'investigations qui se détachent dans l'optique adoptée. Notre objectif principal est de réinsérer les mathématiques et leurs fondements dans l'espace et le temps phénoménaux, à la constitution desquels ces mathématiques contribuent. Notre conviction est, en effet, que grâce à l'espace et le temps nous rétablirons les ponts entre les fondements des mathématiques, qu'on a traditionnellement isolés en leur cherchant un fondement interne (la 
métamathématique de Hilbert est encore une discipline mathématique), et les autres formes de connaissance, comme la physique et les sciences de la vie, dont les phénomènes sont avant tout spatio-temporels. Ce programme épistémologique, un fondement cognitif des mathématiques, commence par l'analyse de notre mode d'accès à l'espace phénoménal. Sans retirer aux analyses logiques formelles leur priorité, en tant que " nécessaires, mais non suffisantes $^{40} »$, nous devons, dans la reconstruction des processus de connaissance par lesquels nous nous arrivons à proposer des concepts et des structures, aller plus loin que l'analyse des seules démonstrations. Ces concepts et structures sont constitués à l'interface entre nous et le monde, à mesure que nous les dessinons sur l'écran des phénomènes dans un effort pour imposer à ce monde un ordre mathématiquement intelligible. Ils ont leur origine dans les régularités que nous "voyons », en tant qu'êtres vivants dans l'histoire, et ils se développent à travers cette histoire par l'intersubjectivité et le langage. Toute l'objectivité et l'efficacité des mathématiques tiennent dans ce processus.

Ces considérations sur le défi de la biologie aux mathématiques n'entendent pas se limiter à des réflexions à caractère informel ou technique, mais elles débouchent sur une analyse de l'interface par laquelle la vie se présente à nous. L'analyse mathématique des problèmes soulevés ici devrait stimuler l'investigation fondationnelle des outils mobilisés pour leur solution : le rôle constituant des mathématiques à l'égard de la réalité en ressortira d'autant mieux que ces problèmes découlent du fait que « autonomie », « critique », " multi-échelle » ne signifient pas la même chose pour les phénomènes de la vie et ceux de la physique. Comme toute analyse de l'interface entre nous et les phénomènes, quelle que soit la forme de connaissance ou mode d'accès à la réalité considéré, cette analyse aura un caractère fondationnel. En termes husserliens, les mathématiques sont une composante clé de «constitution phénoménale » qui a lieu au cœur de chaque analyse de la connaissance.

On se gardera de voir une nouvelle monomanie de la géométrie dans notre insistance sur l'espace, car nous voulons seulement enrichir les paradigmes en vigueur en y réintégrant ce que, pour de bonnes raisons sans doute, en ont exclu les pères fondateurs. D'autant que les mathématiques de l'espace et du temps, étant thèmes transversaux pour plusieurs sciences, des considérations fondationnelles et méthodologiques de ce genre devraient être une composante essentielle de toute recherche interdisciplinaire. S'il nous faut être monistes, ce n'est pas de la méthode, car des phénomènes différents peuvent exiger des outils différents, mais de l'objet. Cela étant, une réflexion explicite sur les différences et

${ }^{40}$ Weyl, 1927. 
analogies méthodologiques peut aussi conduire à une unification, affaire non de transfert ni de superposition de techniques, mais d'une nouvelle synthèse, y compris mathématique (un projet, certes, à long terme).

\section{Références bibliographiques}

(Les articles de G. Longo sont téléchargeables à l'adresse www.di.ens.fr/users/longo)

Aceto L., Bjorn V., Longo G. (eds.) (2003). The difference between Turing Computability and Concurrent Systems, Special issue, Mathematical Structures in Computer Science, n. 4-5, vol. 13, Cambridge U.P.

Asperti A., Longo G. (1991). Categories, Types and Structures, M.I.T. Press.

Bahsoun J., Fiadero J. and Galmiche D. (eds) (1999). Proof Theory of Concurrent Object-Oriented Programming, Special issue, Mathematical Structures in Computer Science, Cambridge U.P., vol. 9, n. 3.

Bailly F. (1991). L'anneau des disciplines, numéro spécial de la Revue Internationale de systémique, vol. 5, n. 3.

Bailly F., Gaill F., Mosseri R. (1993). Orgons and Biolons in Theoretical Biology, Acta Biotheoretica 41, 3-11.

Bailly F., Gaill F., Mosseri R. (1994). Morphogenèse et croissance biologique : un modèle dynamique simple pour le poumon dans La biologie théorique a Solignac, Edition Polytechnica, 65-94.

Bailly F., Longo G. (2003). Objective and Epistemic Complexity in Biology (en preparation).

Barendregt, H. (1984). The lambda calculus: its syntax and semantics, Revised edition, North Holland.

Berthoz A. (1997). Le sens du mouvement, O. Jacob (english transl., Harvard U.P. ; trad. italiana presso Boringhieri. (voir http://www.dmi.ens.fr/users/longo pour un compte-rendu)

Bottazzini U. (1999). Poincaré, Le Scienze.

Bottazzini U., Tazzioli R. (1995). Naturphilosophie and its role in Riemann's Mathematics, Revue d'Histoire des Mathématiques n. 1, 3-38.

Boi L. (1995). Le problème mathématique de l'espace, Springer.

Brown J.H. (1999). West G. Scaling in Biology, Santa Fe Institute Publication.

Connes A. (1994). Non-commutative Geometry, Academic Press.

Edelman G. (1987). Neural Darwinism, Basic Books.

Edelman G. A (2000). Universe of Consciousness. How Matter Becomes Imagination, Basic Books.

Farge M., Kevlhand N., Perrier V., Goirand E. (1996). Wavelets and Turbulence, Proceedings of the IEEEE, 84, n. 4, p. 639-669.

Frege G., (1873). On a Geometrical Representation of Imaginary Forms in the Plane, in Collected Papers on Mathematics, Logic and Philosophy, McGuiness, B. (ed.), Basil Blackwell, Oxford (1984). 
Frege G. (1884). The Foundations of Arithmetic, (english transl. Evanston, 1980).

Gilbert H. (1992). Horizontal integration and cortical dynamics, Neruron, 9 , p. $1-13$.

Girard J.Y., Lafont Y., Taylor R. (1989). Proofs and Types, Cambridge University Press.

Girard J.-Y. (2001). Locus Solum, Special issue, Mathematical Structures in Computer Science, Cambridge U.P., vol.11, n.3.

Goubault E. (ed.) (2000). Geometry in Concurrency, Special issue, Mathematical Structures in Computer Science, Cambridge U.P., vol.10, n.4.

Heath T.L. (1908). The Thirteen Books of Euclid's Elements, Cambridge Univ. Press.

Heinzmann G. (1998). Poincaré on understanding Mathematics, Philosophia Scientiae (143-160).

Hertz J., Krogh A. and Palmer R. (1991). Introduction to the Theory of Neural Computation.

Hilbert, (1899). Les fondements de la géométrie, (trad. fran., Dunod, 1971).

Husserl E. (1933). The origin of Geometry.

Longo G., Petitot J., Teissier B. (1999). Géométrie et Cognition, un manifeste et projet de recherche, téléchargeable de http://www.di.ens.fr/users/longo.

Longo G. (1999). Mathematical Intelligence, Infinity and Machines: beyond the Gödelitis, Journal of Consciousness Studies, special issue on Cognition, vol. 6, 11-12.

Longo G. (2001). Memory and Objectivity in Mathematics, Le Réel en mathématiques, à paraître.

Longo G. (2001a). Some Topologies for Computations invited lecture, colloque Géométrie au XXème siècle: 1930 - 2000, Paris, September 2001a (to appear).

Longo G. (2002). On the proofs of some formally unprovable propositions and Prototype Proofs in Type Theory Invited Lecture, Types 2000, Durham (GB), Dec. 2000 ; Lecture Notes in Computer Science, vol. 2277, Springer.

Longo G. (2002a). Laplace, Turing and the "imitation game" impossible geometry: randomness, determinism and programs in Turing's test. Invited Lecture, Conference on Cognition, Meaning and Complexity, Univ. Roma II, June 2002. (version française parue dans Intellectica, n. 35,2002$)$.

Nabonnand P. (2000). Les recherches sur l'œuvre de Poincaré, Gazette des Mathématiciens, n. 85, Juillet 2000.

Nabonnand P. (2001). La genèse psycho-physiologique de la géométrie selon Poincaré, à paraître dans La revue d'Histoire des Mathématiques.

Nonnenmacher T.F., Losa G.A., Weibel E.R. (1994). Fractals in Biology and Medicine, Birkhauser.

Petitot J., Varela F., Pachoud B. Roy J.-M. (1999). Naturalizing Phenomenology: issues in contemporary Phenomenology and Cognitive Sciences, (J. Petitot et al., eds) Stanford U.P. 
Petitot J. (2000). Complexité neurodynamique en sciences cognitives, Rapport CREA, Ecole Polytechnique.

Poincaré H.(1902). La Science et l'Hypothèse, Flammarion, Paris.

Poincaré H. (1905). La valeur de la Science, Flammarion, Paris.

Poincaré H. (1908). Science et Méthode, Flammarion, Paris.

Poincaré H. (1913). Dernières Pensées, Flammarion, Paris.

Riemann B. (1854). On the hypothesis which lie at the basis of Geometry (engl. transl. by W. Clifford, Nature, 1873 ; trad. italiana e commenti di R. Pettoello, Boringhieri, 1999).

Tappenden J. (1995). Geometry and generality in Frege's philosophy of Arithmetic, Synthesis, n. 3, vol. 102, March 1995.

Varela F. (1999). The specious Present: A Neurophenomenology of Time Consciousness in Petitot et al.

Weyl H. (1918). Das Kontinuum.

Weyl H. (1927). Philosophy of Mathematics and of Natural Sciences (engl. transl., Princeton University Press, Princeton, New Jersey, 1949). 